\title{
Comparison of Enflurane and Propofol in Electroconvulsive Therapy, a Randomized Crossover Open Preliminary Study on Seizure Duration and Anaesthetic Recovery
}

\author{
Zafer Dogan ${ }^{1}$, Nimet Senoglu ${ }^{2}$, Huseyin Yildiz ${ }^{2}$, Ismail Coskuner ${ }^{2}$, Nadiye Ugur ${ }^{3}$, Elif Biter ${ }^{4}$, Hafize Oksuz ${ }^{2}$
}

Summary: Dogan Z, Senoglu N, Yildiz H, Coskuner I, Ugur N, Biter E, Oksuz H - Comparison of Enflurane and Propofol in Electroconvulsive Therapy, a Randomized Crossover Open Preliminary Study on Seizure Duration and Anaesthetic Recovery.

Background and objectives: Electroconvulsive therapy (ECT) is commonly used for treatment of depression, mania and affective disorders. Anaesthetics for general anaesthesia during ECT should have rapid onset, rapid emerge, not interfere with seizure activity and not shorten seizure duration. The aim of this study is to compare effects of enflurane, a pro-convulsive anaesthetic agent, and propofol on seizure durations, postictal suppression index and recovery times during electroconvulsive therapy.

Methods: Unpremedicated subjects were divided into two groups according to induction of anaesthesia. Patients were induced for ECT with $5 \%$ enflurane in group $E$ and $1.2 \mathrm{mg} \cdot \mathrm{kg}^{-1}$ propofol in group P until loss of consciousness. The durations of electroencephalogram (EEG) and motor seizures, postictal suppression index, time to spontaneous breathing, duration of eye opening, and obeying commands were recorded.

Results: There was no statistically significant difference between the groups regarding motor and EEG seizure times and postictal suppression index on the EEG records. Recovery times (times of starting spontaneous breathing, eye opening, and obeying command) were significantly shorter in group E compared to group P. No nausea or vomiting were observed and no ECG abnormality was noted except transient sinus bradycardia and sinus tachycardia.

Conclusions: Although sufficient seizure for the treatment was provided during enflurane anaesthesia, any additional benefit was not revealed regarding seizure times or postictal suppression index when compared to propofol anaesthesia. On the other hand, recovery times after enflurane anaesthesia were shorter than propofol anaesthesia. However, there is still a need for further study in different ETCO $_{2}$ levels.

Keywords: Electroconvulsive Therapy; General Anesthesia, General; Propofol; Enflurane.

\section{INTRODUCTION}

Electroconvulsive therapy (ECT) is commonly used for treatment of depression, mania and affective disorders. The brain is subjected to seizure activity induced by the electric current, and adequate seizures are necessary for a therapeutic response ${ }^{1,2}$. Although the mechanism of action is not clearly understood, a definite correlation between seizure duration and

Received from Anaesthesiology and Intensive Care, Medical School, Benzmiâlem Vakif Üniversitesi, Turkey.

1. MD; Assist. Prof. Benzmiâlem Vakif Üniversitesi, Faculty of Medicine, Department of Anaesthesiology, Istanbul, Turkey

2. MD; Assist. Prof. Kahramanmara Sütçü mam Üniversitesi, Faculty of Medicine, Department of Anaesthesiology, Kahramanmaras, Turkey

3. MD; Clinical Resident Kahramanmara Sütçü mam Üniversitesi, Faculty of Medicine, Department of Anaesthesiology, Kahramanmaras, Turkey

4. MD; Clinical Resident Kahramanmara Sütçü mam Üniversitesi, Faculty of Medicine, Department of Psychiatry, Kahramanmaras, Turkey

Submitted on January 25, 2011.

Approved on March 14, 2011.

Correspondence to

Dr. Zafer Dogan

Benzmiâlem Vakif Üniversitesi, Faculty of Medicine

Department of Anaesthesiology

Istanbul, Turkey

E-mail:drzdogan@yahoo.com clinical outcome remains to be demonstrated. Because these factors alone are not necessarily a predictor of outcome, adequate seizures are supposed to be more efficacious. Motor seizure duration greater than 25 seconds has been recommended to ensure clinical adequacy of treatment, and short seizures are considered negative predictors of outcome ${ }^{1,3}$.

Anaesthetics, which are used for general anaesthesia during ECT, should have rapid onset, rapid emergence, not interfere with seizure activity, and not shorten seizure duration. Since most short-acting anaesthetics have anticonvulsant effects, they can increase the threshold and inhibit the spread of seizure, thus modifying the seizure activity and shorten its duration 1 .

Propofol is widely used in ECT anaesthesia as a reference agent due to characteristics such as rapid onset and emergence from anaesthesia, minimal postoperative confusion and a lower incidence of hypertension or tachycardia during induction of anaesthesia. However, it produces a dose-dependent decrease in seizure duration ${ }^{1,3}$.

Enflurane is a volatile anaesthetic that could be used for induction of anaesthesia ${ }^{4-6}$. Enflurane, unlike other commonly used inhalation anaesthetics, may precipitate seizure activity on the electroencephalogram with high amplitude spike wave complexes with burst suppression. Even in some patients, 
convulsive myoclonic movements during anaesthesia have been observed ${ }^{7-11}$. There is not any trial in PubMed about enflurane anaesthesia during ECT procedure.

The aim of this study was to compare the effect of enflurane, a proconvulsive anaesthetic agent, and propofol, as a reference anaesthetic agent, on seizure durations, haemodynamic status, postictal suppression index, and recovery times after electroconvulsive therapy.

\section{METHODS}

After the Faculty Ethics Committee approval, patients scheduled by Psychiatry Clinic for ECT were enrolled for second to fifth ECT. Written informed consent was provided from the patients who were able to provide the consent. The trial was conducted as a prospective open technique. Exclusion criteria included mask intolerance, seizure disorder, obesity (body mass index $\geq 30$ ), gastroesophageal reflux disease resistant to pharmacotherapy, current alcohol or other substance usage, unstable cardiovascular disease, chronic obstructive pulmonary disease, renal or hepatic failure, and organic brain disease.

Ten patients with major depression were included the study. No patient was premedicated. Randomization was performed by a consultant anaesthesiologist using a sealed envelope technique before the first session because of the crossover design. The subjects were divided into two groups according to induction anaesthetics of second ECT. At the first session, suprathreshold electrical stimulus was detected and then, two agents were alternated over the remaining four sessions of ECT as crossover design. After routine monitorization and preparation, general anaesthesia was induced with $5 \%$ enflurane in group $E$ and $1.2 \mathrm{mg} \cdot \mathrm{kg}^{-1}$ propofol in group $P$ until loss of consciousness. Loss of consciousness was defined as both loss of the eyelid reflex and no response to the verbal command, e.g., "open your eyes". After loss of consciousness, enflurane was firstly lowered to $2 \%$ in group E. No additional propofol was needed to assure loss of eyelid reflex. After loss of consciousness, a pressure cuff was applied to the arm and inflated above the systolic arterial pressure to isolate the circulation of the arm, and to permit an accurate assessment of the motor seizure. Muscle relaxation was achieved with succinylcholine $0.5 \mathrm{mg} \cdot \mathrm{kg}^{-1}$. After resolution of muscle fasciculation, enflurane was stopped in group $E$.

A bite blocker was inserted before the session, and ventilation was assisted manually using a face mask with $100 \%$ oxygen at 6 L. $\mathrm{min}^{-1}$ fresh gas flows in all groups. The patients were maintained in normocapnia. After the anaesthesia for ECT in group E, anaesthetic system was cleared by fresh oxygen.

Noninvasive mean arterial pressure (MAP) and heart rate (HR) values were recorded before induction of anaesthesia (baseline), one minute after induction, and one, five and ten minutes after ECT. Just before ECT, end-tidal $\mathrm{CO}_{2}$ and endtidal enflurane concentration in the last breath were recorded using Cardiocap 5 monitor (Datex Ohmeda, Louisville, USA).

Before the trial, each patient's ECT-induced seizure threshold was determined by administering successive stimuli of in- creasing intensity at 30 -second intervals until a generalized motor seizure was induced (threshold stimulus) in the first session. The suprathreshold stimulus (one level higher intensity than the threshold stimulus) was used throughout the study and was maintained constantly for each patient.

The suprathreshold electrical stimulus was delivered via bifrontotemporal electrodes using a Thymatron System IV machine (Somatics, Inc., Lake Bluff, IL, USA) by the referring psychiatrist immediately after resolution of muscle fasciculation. The duration of seizure was recorded as the time from the ECT stimulus to cessation of electroencephalogram (EEG) activity. Motor seizure activity was recorded according to the cuff method defined above. Postictal suppression indexes on EEG records, time of spontaneous breathing, eye opening, and obeying commands were recorded.

Assuming an alpha level of 0.05 with a power of 0.80 , a minimum of 17 data in each group were required to detect a mean difference of $5 \mathrm{sec}$ and standard deviation of $5 \mathrm{sec}$ for the seizure duration between the two groups. The statistical analysis of data was performed with SPSS 11.0 (SPSS Inc., Chicago, IL, USA). Data are expressed as mean \pm SD or absolute values. The independent-sample t-test was used for comparisons between groups. Data were analyzed using repeated-measures analysis of variance with Bonferroni correction. A value of $p<0.05$ was considered as significant.

\section{RESULTS}

Ten patients, four male and six female, with a mean age of $36.80 \pm 11.14$ years (range 23 to 59 ) and weight of $79.60 \pm 14.98 \mathrm{~kg}$ (range 62 to 95 ) received a total of fourty ECT sessions. Hemodynamic variables are shown in Table I. Seizure data and recovery times after ECT in both groups are shown in Table II.

Mean arterial pressure and heart rate were significantly higher in group $\mathrm{E}$ only at one min after induction. On the other hand, mean arterial pressure and heart rate were significantly higher in both groups at only one min following ECT when compared to baseline values. Also in group $\mathrm{E}$, heart rate at one min after induction was significantly higher compared to baseline value. Haemodynamic values at baseline, five and ten min after ECT, were similar in both groups (Table I).

There were no significant differences between groups regarding motor and EEG seizure times and postictal suppression index on the EEG records (Table II).

Recovery times (times of starting spontaneous breathing, eye opening, and obeying command) were significantly shorter in group $\mathrm{E}$ with powers of $96 \%, 100 \%$, and $100 \%$, respectively (Table II). No nausea or vomiting was observed and no ECG abnormality was noted, except transient sinus bradycardia and sinus tachycardia.

\section{DISCUSSION}

There are multiple factors affecting the choice of anaesthesia in ECT, including seizure length, hemodynamics, and recovery times. 
Table I - Hemodynamic Values

\begin{tabular}{llll}
\hline & Group $P(\mathrm{n}=20)$ & Group $\mathrm{E}(\mathrm{n}=20)$ & $\mathrm{p}$ \\
\hline Heart Rate $(\mathrm{bpm})$ & & & 0.314 \\
Baseline & $86.80 \pm 5.44$ & $85.05 \pm 7.26$ & 0.016 \\
1 min after induction & $89.70 \pm 4.09$ & $97.05 \pm 12.35^{\star} \S$ & 0.438 \\
1 min after ECT & $105.25 \pm 7.84 \S$ & $102.90 \pm 10.86 \S$ & 0.953 \\
5 min after ECT & $88.85 \pm 7.62$ & $88.70 \pm 8.32$ & 0.056 \\
10 min after ECT & $88.25 \pm 3.38$ & $85.80 \pm 4.39$ & 0.695 \\
MAP (mmHg) & & & 0.033 \\
Baseline & $90.60 \pm 5.15$ & $89.90 \pm 6.01$ & 0.310 \\
1 min after induction & $90.50 \pm 9.39$ & $96.65 \pm 8.10^{*}$ & 0.870 \\
1 min after ECT & $104.00 \pm 12.76 \S$ & $107.25 \pm 6.03 \S$ & 0.688 \\
5 min after ECT & $97.10 \pm 19.12$ & $97.90 \pm 10.45$ & \\
10 min after ECT & $94.50 \pm 8.53$ & & \\
\hline
\end{tabular}

n: number of ECT sessions, ${ }^{*} p<0.005$ when compared to propofol group, $\S p<0.005$ when compared to baseline value, ECT: electroconvulsive therapy.

Table II - Duration of Seizures and Recovery Profile

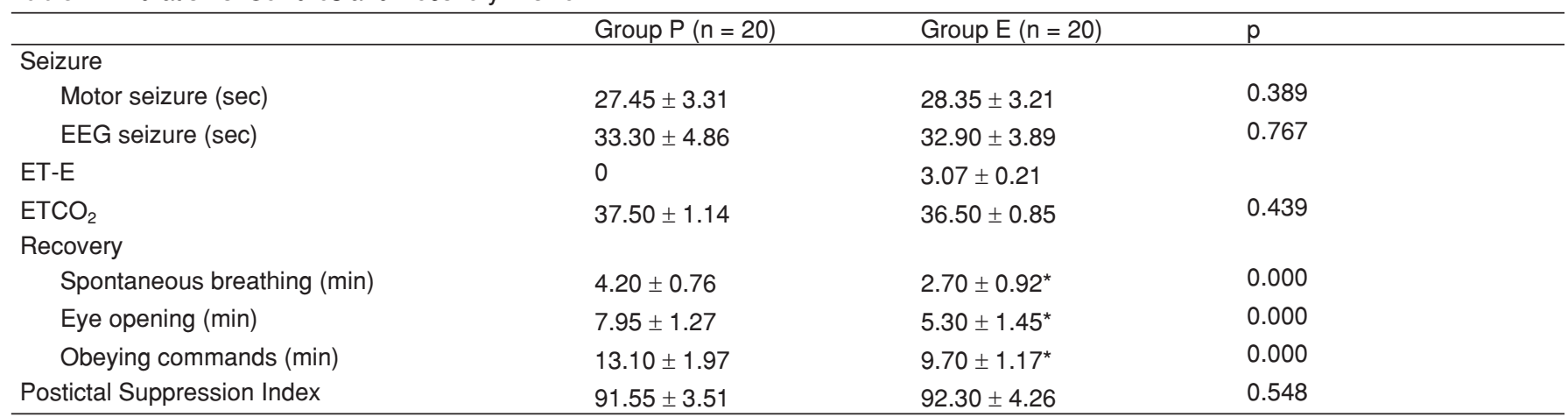

${ }^{*} \mathrm{p}<0.005$ when compared to propofol group, EEG: electroencephalogram, ET-E: end-tidal enflurane concentration, ETCO ${ }_{2}$ : end-tidal carbon dioxide concentration.

Induction of anesthesia could be provided by mask anesthesia in patients with needle-phobia in whom multiple attempts are required for successful placement of the intravenous catheter because of agitation or obesity ${ }^{12}$. Mask anesthesia with sevoflurane was performed in ECT procedure and favourable results were reported ${ }^{12-16}$. Enflurane is another volatile anesthetic that could be used in anesthesia induction in children ${ }^{4}$ and adult 5,6 patients.

Unlike most other anaesthetics, previous animal 17 and epileptic human ${ }^{10}$ studies and normal human reports ${ }^{7}$ have shown a high incidence of spike activity on EEG and grand mal seizure patterns induced by enflurane. The findings with enflurane have also been confirmed in patients with temporal lobe epilepsy during both electrocorticographic and depth electrode recordings. In ten healthy volunteers, grand mal seizure patterns were precipitated by auditory, visual, and tactile stimulation at end-tidal enflurane concentrations of $3 \%-6 \%$. With an alveolar enflurane concentration between $2.5 \%$ and $3 \%$, hyperventilation to an average $\mathrm{PaCO}_{2}$ of 22 torr $(\mathrm{mmHg})$ produced an increase in the frequency, magnitude, and synchrony of the spiking activity in epileptics ${ }^{7}$. This technique has since been used to activate silent epileptogenic foci intraoperatively, to delineate the site of seizure activity before discrete surgical excision ${ }^{11}$. So it was presumed that enflurane may increase duration of convulsion during ECT and no literature was found in PubMed about enflurane for ECT. In this paper, propofol was a reference anaesthetic agent used in many anaesthesia clinics for ECT.

The result revealed that enflurane anaesthesia assures satisfactory motor and EEG seizures. But there was no significantly difference in motor and EEG seizure times compared to propofol anaesthesia. Because enflurane is known to increase seizure activity, it is expected that seizure durations are longer than in propofol group. This could be explained by the epileptic activity associated with enflurane anaesthesia, which was particularly likely to occur with high concentrations of enflurane and in the presence of hypo- or hypercapnia ${ }^{7,9,11}$. Ventilation in this study was maintained via face mask manually assisted in order to obtain normal ETCO level, so that hypo- or hypercapnia were not allowed. Hypocapnia might have increased the duration of convulsions ${ }^{18}$; however, it would be technically difficult to provide and to detect exactly by mask ventilation, and may affect haemodynamics and perfusion. On the other hand, this effect was shown during sevoflurane anaesthesia, and seen only in the first session of treatment in that study ${ }^{18}$. The relationship between enflurane concentration, $\mathrm{PaCO}_{2}$, 
and epileptiform activity was evaluated in a volunteer study ${ }^{7}$. It was demonstrated that the minimum epileptogenic enflurane concentration was approximately $1 \%$ lower when $\mathrm{PaCO}_{2}$ was 20 torr (vs normocarbia), and $1 \%$ higher when $\mathrm{PaCO}_{2}$ was 60 torr.

Hemodynamic variables during ECT depend on generalized autonomic nervous system stimulation, with an initial parasympathetic-induced bradycardia lasting 10 to 15 seconds, followed immediately by a more prominent sympathetic response. Therefore, blood pressure and HR are transiently increased by $20-30 \%$ or more ${ }^{19}$. In this study, haemodynamic values were not different except at the first minute after anaesthetic induction between the groups congruently. Enflurane represents an intermediate values between isoflurane and sevoflurane with respect to the circulatory changes ${ }^{6}$. On the other hand, there was a haemodynamic compromise at the first minute after ECT in both groups when compared to baseline value in our study. In a previous study ${ }^{13}$, there were significant elevations in sevoflurane group after one and ten minutes ECT, but these elevations were not seen in $1.5 \mathrm{mg} \cdot \mathrm{kg}^{-1}$ of propofol group. This was probably a result of slow increasing and rapid decreasing the alveolar concentration of volatile anaesthetics with respiration. In the current study, propofol dose was lowered to $1.2 \mathrm{mg} . \mathrm{kg}^{-1}$ in order to not decrease seizure durations. This could be a reason for tachycardia at the first minute after ECT procedure.

In addition, mean arterial pressure and heart rate were significantly higher in group $\mathrm{E}$ only at one min after induction when compared to group $\mathrm{P}$. This could be probably a result of mask induction. During mask induction in the first minute, because patients were not exactly unconscious yet, this could be considered as an acceptable reaction. On the other hand, in Toprak's study ${ }^{13}$, in sevoflurane group at the first min after induction this reaction was not seen. This could be explained with blood-gas partition coefficient that is 0.65 and 1.5 for sevoflurane and enflurane respectively 20 .

Although there is not exact relationship between postictal suppression and outcome, greater postictal suppression was accepted to be associated with favourable ECT outcome ${ }^{21}$. In this study, not only seizure time but postictal suppression index in group $E$ was acceptable level, but not significantly different compared to group P as well. Gazdag et al. ${ }^{22}$ determined that postictal suppression index of propofol was $86 \%$.
Because of shortness of ECT procedure an anaesthetic agent that has rapid recovery should be of choice. In this respect, succinylcholine is preferred as neuromuscular blocker. Otherwise, succinylcholine is not effective on seizure duration, post-seizure recovery and hemodynamics ${ }^{12}$. For rapid recovery, propofol is preferred in our clinic for ECT sessions. Methohexital was approved as another reference anaesthetic agent for ECT ${ }^{19}$. In our country, methohexital is not established for routine use.

In Toprak's study ${ }^{13}$ propofol's recovery profile was similar to sevoflurane's. Inhalation anaesthetics could not be stored in lipose tissue in this short time. After discontinuation of inhalation anaesthetics, they are exhaled from lungs rapidly, and then blood concentration of inhalation anaesthetics is too low to unconsciousness and to assure anaesthesia. In order to assure the anaesthesia enflurane was not stopped just lowered in group E. Recovery times of patients (starting spontaneous breathing, eye opening, and obeying commands) were shorter in enflurane group than in propofol group in our study. These were also probably results of rapid decreasing the alveolar concentration of enflurane by respiration and/or residual effect of propofol. Because we lowered to $2 \%$ of enflurane after loss of conscious, it could be another reason of this rapid recovery. In Toprak's study sevoflurane was inhaled at $7 \%$, but we do not know what they did after loss of conscious.

The present study has several limitations. Enflurane is an obsolete anaesthetic agent. Proconvulsive property of enflurane guided the study, and the convulsive effect of enflurane must have been evaluated during ECT in this aspect. On the other hand, the trial had been designed as open trial, because of the drugs' nature used. Moreover, as this was a preliminary study, the number of patient may not be sufficient for an accurate outcome.

In conclusion, although high incidence of spike activity on EEG and grand mal seizure patterns could be induced by enflurane, motor and EEG seizure times or postictal suppression index were not increased by enflurane in the presence of normocapnia when compared to propofol anaesthesia. Although enflurane anaesthesia provided enough seizure for ECT, it did not provide additional beneficial effect regarding seizure times. In addition, recovery times were shorter than with propofol anaesthesia. However, there is still need for further study in different $\mathrm{ETCO}_{2}$ levels. 